\title{
Pomegranate: High Binding Affinity for PPARr, a Drug Target for Diabetes Type 2, and Lipid Remodelling in Adipocytes
} Svjetlana Medjakovic ${ }^{2}$, Stefanie Hobiger ${ }^{2}$ and Alois Jungbauer ${ }^{1 *}$

${ }^{1}$ Department of Biotechnology, University of Natural Resources and Life Sciences Vienna, Muthgasse 18, 1190 Vienna, Austria ${ }^{2}$ Christian-Doppler-Laboratory of Receptor Biotechnology, Muthgasse 18, 1190 Vienna, Austria

\begin{abstract}
The remodeling of lipids in adipocytes is an interesting property of compounds with the ability to ameliorate metabolic syndrome. In this study, the binding affinity of pomegranate to the peroxisome proliferator-activated receptor $\gamma$ (PPARY) was compared with plant extracts that are commercially on offer as anti-diabetic dietary supplements such as cinnamon, purslane, grape wine, bitter melon, Kothala himbutu, and Coccinia indica. This receptor is a drug target for diabetes type 2. One of the most potent pomegranate extracts was selected and tested in a murine 3T3-L1 cell system to examine effects on adipocyte differentiation. Pomegranate extracts had an extremely high binding affinity for PPARy, which was several fold higher in comparison to other tested extracts. Pomegranate extract also modulated adipocyte differentiation, which resulted in lipid remodeling and the formation of micro-lipid droplets, which increases total lipid droplet surface area to volume ratio and enables an easier lipid breakdown due to lipolysis.
\end{abstract}

The results corroborate the hypothesis that pomegranate has a benevolent impact on obesity and diabetes type 2. As modulator of PPARy and the adipose tissue, pomegranate is a promising plant for the amelioration of metabolic syndrome.

\section{Keywords: Adipocytes; PPAR $\gamma$; Diabetes type 2}

\section{Introduction}

Cellular free fatty acids are toxic for cells, which also contributes to the pathogenesis of cardiovascular diseases and metabolic syndrome. Cells handle so-called lipotoxicity resulting by an excess of free fatty acids via triglyceride accumulation [1]. This happens not only in adipocytes, but in all cells; the amount and size of lipid droplets in adipocytes is much bigger, as these cells are meant to function as lipid storage of the organism. Meanwhile is the triglyceride accumulation in non-adipose tissue a consequence of an acute free fatty acid excess and represents a defence mechanism against lipotoxicity. The results from Paar et al.[2] which show a de novo synthesis of micro Lipid Droplets (mLDs) during lipolysis, implicate that mLD formation could not only pose as facilitation of lipolysis by increasing the working surface for lipases, but protects also cells from lipotoxicity by relocation of free fatty acids into lipid droplets. The formation of mLDs is linked with the ability of the cell to re-esterify fatty acids. When this is blocked, mLDs cannot be generated [3]. mLDs are active sites of lipolysis [3] and compounds that promote the formation of $\mathrm{mLDs}$ are potential drugs for the treatment of obesity and metabolic syndrome.

Obesity, diabetes type 2 and metabolic syndrome are a growing problem, which is increasing every year. It has the potential to get pandemic in the next decades. By now, obesity and overweight are linked to more deaths worldwide than underweight [4]. And metabolic syndrome is on the rise. This is no longer only a problem of Western world.

Diabetes type 2 is a lifestyle disease. Lifestyle changes that include body weight reduction, a healthier diet and regular exercise can prevent diabetes initiation but also progression. The diet should be switched to a diet that is rich in nutrients and low in saturated and trans fats, while rich in monounsaturated and polyunsaturated fats and low in calories. The diet should also include a lot of fruits and vegetables, as these contain not only vitamins but also a lot of bioactive compounds.

Pomegranate is a fruit that is very suitable for the prevention of obesity, diabetes and metabolic syndrome. To go one step further, pomegranate is also suitable as diabetes co-treatment; several studies support the ethnomedical use of pomegranate in diabetes treatment and draw the picture of a plant that is hypoglycemic, able to increase insulin sensitivity and improves blood lipid parameters. The emphasis is on co-treatment rather than treatment alone; in form of dietary supplements, pomegranate may assist prescribed diabetes medication. If a potent single compound may be isolated from pomegranate, it could result in a new anti-diabetic drug. Metformin, one of the most commonly prescribed drugs in diabetes therapy, has its origin in French lilac, also called goat's rue. But if such an isolation of an active anti-diabetic compound is possible from pomegranate, remains to be seen.

Anti-diabetic drugs can act through various mechanisms. Just recently Mazzola [5] summarized therapy possibilities of type 2 diabetes mellitus. Classical therapy is focused on $\beta$-cell impairment and insulin resistance. Some anti-diabetics can lead to weight gain. This is true for insulin, sulfonylureas, glinides and glitazones. The latter ones increase insulin sensitivity via the PPAR $\gamma$ pathway, but have beside weight gain also other serious side effects. Because of that, troglitazone and rosiglitazone were withdrawn from the market or the access was restricted. Safety of pioglitazone has been discussed recently in light of a putative increase of bladder cancer [6,7] and could be the next glitazone drug to be withdrawn.

Given the current situation regarding PPAR $\gamma$ modulating drugs, there is definitely a need for new drugs that act as PPAR $\gamma$ ligands. Solution to this problem could represent ligands that simultaneously activate all PPAR isoforms, so-called pan-PPAR activators. They have been discussed as novel therapeutic approach to treat metabolic

${ }^{*}$ Corresponding author: Alois Jungbauer, Department of Biotechnology, University of Natural Resources and Life Sciences Vienna, Muthgasse 18, 1190 Vienna, Austria, Tel: 0043-1-476-546-226; Fax: 0043-1-476-546-675; E-mail: alois.jungbauer@boku. ac.at

Received April 09, 2014; Accepted May 20, 2014; Published May 22, 2014

Citation: Medjakovic S, Hobiger S, Jungbauer A (2014) Pomegranate: High Binding Affinity for PPARy, a Drug Target for Diabetes Type 2, and Lipid Remodelling in Adipocytes. Nat Prod Chem Res 2 :136. doi:10.4172/2329-6836.1000136

Copyright: (c) 2014 Medjakovic S, et al. This is an open-access article distributed under the terms of the Creative Commons Attribution License, which permits unrestricted use, distribution, and reproduction in any medium, provided the original author and source are credited. 
syndrome [8]. Pomegranate and compounds thereof have been reported to modulate PPAR $\alpha, \beta$ and $\gamma$ [9-13].

\section{Material and Methods}

\section{Chemicals and media}

Dimethylsulfoxide (DMSO) was obtained from Sigma-Aldrich (St. Louis, MO) and rosiglitazone from Cayman Chemicals (Ann Arbor, MI, USA). Extract powders were provided by Apomedica Pharmazeutische Produkte GmbH (Graz, Austria) who received them from contract extract manufacturers. Polar Screen ${ }^{\mathrm{TM}}$ PPAR Competitive Assay was purchased from Invitrogen Corporation (Carlsbad, CA, USA).

DMEM media (without Na-pyruvate, with $3.7 \mathrm{~g} / 1 \mathrm{NaHCO}_{3}$, with $4.5 \mathrm{~g} / \mathrm{l} \mathrm{D}$-glucose, without L-glutamine and with phenol red), L-alanylL-glutamine (200 $\mathrm{mM}$ stock solution), Phospho Buffered Saline (PBS) solution (without $\left.\mathrm{Ca}^{2+}, \mathrm{Mg}^{2+}\right)$, trypsin ((1:250)/EDTA (0.25\%/0.02\%) in PBS, without $\left.\mathrm{Ca}^{2+}, \mathrm{Mg}^{2+}\right)$ and heat-inactivated fetal bovine serum (FBS) were purchased from Biochrom (Berlin, Germany). Bovine Serum (BS) was obtained from Invitrogen Corporation (Carlsbad, CA, USA).

3-Isobutyl-1-methylxanthine (IBMX), Dexamethasone (DEX) and insulin (from bovine pancreas, $10 \mathrm{mg} / \mathrm{mL}$ in $25 \mathrm{mM}$ HEPES, $\mathrm{pH}$ 8.2), formalin (10\%), Oil Red O, and isopropanol were purchased from Sigma-Aldrich (St. Louis, MO).

\section{Polar screen PPAR competitive assay}

The PPAR ligand-binding competitive assay was performed according to the manufacturer's protocol. Briefly, the PPAR $\gamma$ LigandBinding Domain (LBD) and the fluorescent PPAR $\gamma$ ligand form a complex with a high polarization value. Upon displacement of the fluorescent ligand by other ligands, the fluoromone is set free and the polarization value decreases. This is concentration dependent and is used to determine the relative binding affinity of test compounds for PPAR $\gamma$. Fluorescence polarization was measured with a Genios Pro plate reader (Tecan, Crailsheim, Germany) at an excitation wavelength of $485 \mathrm{~nm}$ and an emission wavelength of $535 \mathrm{~nm}$. Rosiglitazone (DMSO solution), a prototypical PPAR $\gamma$ ligand, was used as reference compound.

For the calculation of $\mathrm{IC}_{50}$ values, extracts were tested in different concentrations from $50 \mathrm{mg} / \mathrm{ml}$ to $1 \mathrm{ng} / \mathrm{ml}$ (final concentration of 1.25 $\mathrm{mg} / \mathrm{ml}$ to $0.025 \mathrm{ng} / \mathrm{ml}$ ), depending on the optimal concentration range, which was evaluated in preliminary test runs.

Data for the $\mathrm{IC}_{50}$ evaluation were obtained from at least three independent experiments.

\section{Equations and calculations}

Decreasing polarization values due to displacement of the fluorescent ligand are a measure for the binding affinity of the test compound to PPAR $\gamma$. Polarization values were plotted against the concentration of the extracts (logarithmic scaling), fitted using a logistic dose-response function and $\mathrm{IC}_{50}$ values were calculated (Table Curve 2D software (Jandel Scientific)).

The logistic dose-response curve is described in equation 1.

$$
\mathrm{Y}=\mathrm{a}+\frac{\mathrm{b}}{1+(\mathrm{c} / \mathrm{x})^{\mathrm{d}}}
$$

where $\mathrm{a}$ is the baseline, $\mathrm{b}$ is the plateau of the curve designated as the ligand efficiency, and $c$ gives the transition center which is the concentration that displaces $50 \%$ of the fluorescent ligand. Parameter $\mathrm{d}$ is the transition width, $\mathrm{Y}$ is the polarization units, and $\mathrm{x}$ is the concentration. The sum of parameters $a$ and $b$ of the reference compound rosiglitazone was used to normalize data to the maximum efficiency.

The mean $\mathrm{IC}_{50}$ value (of three independent experiments) of an extract and the mean $\mathrm{IC}_{50}$ value of rosiglitazone (all experiments) were used to calculate the equivalent concentration of the extracts (equation 2).

Equivalent concentration $[\mathrm{nmol} / \mathrm{g}]=\frac{\overline{\mathrm{x}}_{\mathrm{R}}\left(\mathrm{IC} 50_{\text {Rosiglitzone }}\right)[\mathrm{nmol} / \mathrm{L}]}{\overline{\mathrm{x}}_{\mathrm{E}}\left(\mathrm{IC} 50_{\text {Extract }}\right)[\mathrm{g} / \mathrm{L}]}$

\section{Extract preparation}

Dry extract powders were stirred in DMSO with a concentration of $50 \mathrm{mg} / \mathrm{ml}$ at room temperature for $24 \mathrm{~h}$. Extracts were centrifuged at $13,200 \mathrm{rpm}$ and room temperature. The clear supernatant was stored at $-20^{\circ} \mathrm{C}$ until use. Extracts were diluted in DMSO for further testing.

\section{Cell culture maintenance}

3T3-L1 pre-adipocytes were obtained from the American type Culture Collection and were maintained at $37^{\circ} \mathrm{C}$ under a humidified $5 \% \mathrm{CO}_{2}$ atmosphere. Cells were grown in DMEM medium and supplemented with $4 \mathrm{mM}$ L-alanyl-L-glutamine and $10 \%$ bovine serum (sterile filtered). Cells were subcultured once or twice a week when they reached $\sim 80-90 \%$ confluency.

\section{Adipocyte assay}

3T3-L1 pre-adipocytes were seeded in 96 well plates (sterile, transparent, flat bottom) from Nunc (Roskilde, Denmark) with $5 \times 10^{4}$ cells/well in DMEM with $10 \%$ BS. After two days, cells reached confluency (day 0). At day 2, cells were cultured in induction media comprising DMEM with 10\% FBS and supplemented with $0.5 \mathrm{mM}$ IBMX and $1 \mu \mathrm{M}$ DEX. Pomegranate extract (2) was added at $1 \%$. Rosiglitazone was added at a final concentration of $0.1 \mu \mathrm{M}$ as positive control. Negative controls were media only and wells with DMSO (1\%). On day 5 and 7, media was replaced by progression media (DMEM with $10 \% \mathrm{FBS}$, supplemented with $10 \mu \mathrm{g} / \mathrm{ml}$ insulin). Extract and controls were added again as previously described. On day 9, lipid droplets of the differentiated adipocytes were stained with Oil red O solution.

\section{Oil red $O$ staining}

After differentiation, the cells were washed with PBS and fixed with $10 \%$ formalin in PBS for 15 minutes at room temperature, and washed twice with washing solution (60\% isopropanol). After wells dried, the cells were stained with filtered Oil red $\mathrm{O}$ working solution ( $0.36 \%$ in $60 \%$ isopropanol, freshly prepared prior to use from a $0.6 \%$ solution in pure isopropanol and filtered with $0.2 \mu \mathrm{m}$ ) for 20 minutes at room temperature. Then wells were washed with distilled water until it was clear and photographed (Olympus DP20) under the microscope (Olympus CKX41). Additionally the lipid droplets were measured with the distance measurement function of the camera. At least thirty droplets were gauged and the distribution of lipid droplets sizes was evaluated.

\section{Results and Discussion}

We have screened extracts for PPAR $\gamma$ affinity because this could be a key to predict lipid remodeling in adipocytes.

\section{PPAR $\gamma$ binding affinity}

Pomegranate extracts had the highest binding affinity to PPAR $\gamma$. 
Some of the tested extracts were delivered with a high drug-extract ratio. Hence, the results were evaluated twice: in Figure 1 the total effect of the extract as it is offered, marketed and eventually consumed, is presented. As the drug extract ratio specifies the amount of drug that was used for the extract preparation, it means if an extract has a drug extract ratio of 75:1 that $75 \mathrm{~g}$ of the plant were collected, extracted and dried to $1 \mathrm{~g}$ of the extract. This means that compounds that are in all likelihood the bioactive ingredients have been extremely enriched. The test results were in case of a 75:1 drug extract ratio divided by 75 to take the drug extract ratio into account Figure 2. This gives information about how potent the plant (part) really is, without enrichment.

As can be seen in Figure 2, all pomegranate extracts were more potent than all other tested extracts that are commercially available as diabetes remedies such as cinnamon, purslane, grape wine, bitter melon, Kothala himbutu, and Coccinia indica. Additional information about the extracts can be found in Table 1 . The equivalent concentration of extracts (1) pomegranate whole fruit, (2) pomegranate fruit, (3) pomegranate fruit, (4) pomegranate peel, (5) pomegranate whole fruit, (6) pomegranate, (7) grape wine, (8) ginseng, (9) Kothala himbutu, (10) purslane, (11) bitter melon, (12) cinnamon bark, and (13) cinnamon bark, was 2701 561.9, 1154 646.2, 483 095.6, 265 279.7, 206 211.1, 103 318.7, 25 551.0, 9 586.9, 5 922.1, 4 814.2, 1 511.3, 1 042.6, and $1084.2 \mathrm{nmol}$ rosiglitazone per gram sample, respectively. Extract (14), Coccinia indica, showed within the tested concentration range in the assay, no binding affinity.

The order of potency changed when in the evaluation the drug extract ratio was taken account of Figure 2. Still, pomegranate extracts were the most potent extracts, although grape wine was among the top five extracts in this tabulation form. The ranking of the extracts changed also within the top five. In addition, pomegranate peel seems to contain less active compounds than the whole fruit or the fruit only, as the binding affinity of this extract was much lower compared to fruit or whole fruit extracts. But as only one pomegranate peel extract was tested, this may not be a significant observation.

Extracts (1) and (2) had the highest binding affinity to PPAR $\gamma$ in total, but when the drug extract ratio was considered, extract (1) was not the first in the ranking, but the fourth. This is due to the extreme enrichment of this extract with an extract drug ratio of 75:1. As it is listed in Table 1 as additional information, extract (1) contains $80 \%$

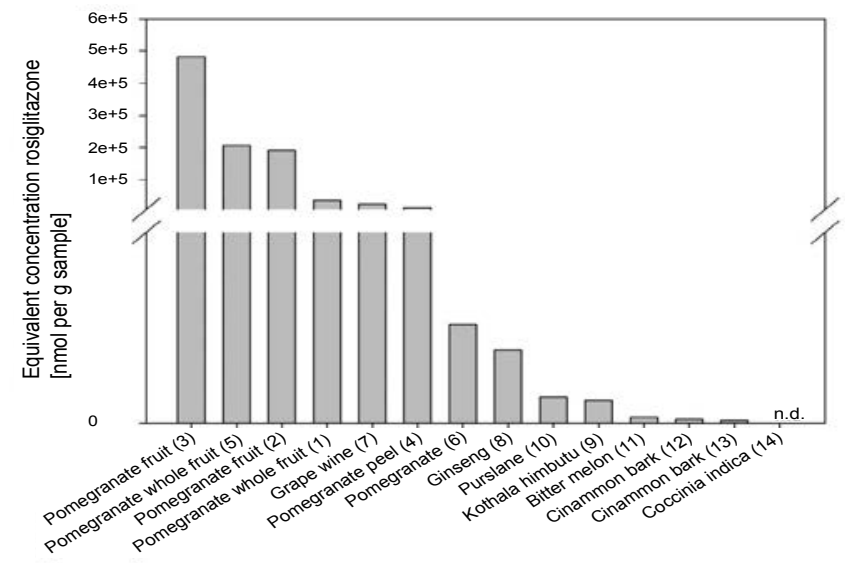

Figure 1: Equivalent concentration, given as nmol rosiglitazone per gram sample, of extracts compared to reference compound rosiglitazone in the PPAR $\gamma$ ligand-binding competitive assay. Abbreviation: n.d., no binding to PPAR $\gamma$ detected in the assay.

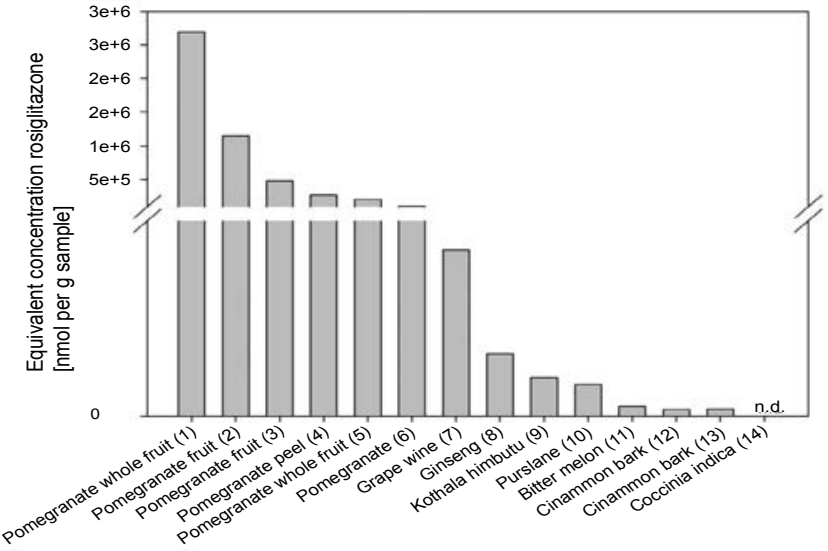

Figure 2: Equivalent concentration of extracts compared to reference compound rosiglitazone in the PPAR $\gamma$ ligand-binding competitive assay taking the drug extract ratio (see Table 1 ) into account and given as nmol rosiglitazone per gram sample. Abbreviation: n.d., no binding to PPAR $\gamma$ detected in the assay.

polyphenols. Unfortunately the producer does not specify which polyphenols those are. Extract (2) is the second potent extract in total. The drug extract ratio is only 6:1 and it loses only one place in the ranking, when the drug extract ratio is included in the evaluation. When the drug extract ratio of all extracts was included in the evaluation, the equivalent concentration of (1) pomegranate whole fruit, (2) pomegranate fruit, (3) pomegranate fruit, (4) pomegranate peel, (5) pomegranate whole fruit, (6) pomegranate, (7) grape wine, (8) ginseng, (9) Kothala himbutu, (10) purslane, (11) bitter melon, (12) cinnamon bark, and (13) cinnamon bark, was 36 020.8, 192 441.0, 483 095.6, 13 264.0, 206 211.1, 2 583.0, 25 551.0, $1917.4,592.2,687.5,151.1$, 104.3 , and $72.3 \mathrm{nmol}$ rosiglitazone per gram sample, respectively.

Pomegranate (Punica granatum) or compounds thereof have been reported as $\operatorname{PPAR} \gamma$ modulators previously [14,15,12]. In this study, it was evaluated how potent pomegranate, a plant that has been described as putative anti-diabetic, is compared to plants that are already commercially available as anti-diabetes remedies or dietary supplements. This was evaluated with the binding affinity to PPAR $\gamma$, a drug target for a class of anti-diabetes agents.

All pomegranate extracts that have been tested in this study, had by far much higher binding affinities for PPAR $\gamma$ than all other tested extracts. It has been reported that pomegranate exerts hypoglycemic effects, increases insulin sensitivity, inhibits $\alpha$-glucosidase, modulates glucose transporter type 4 (GLUT4) function and improves blood lipid parameters (reviewed [16]). Several, but not all, of these actions can be linked to PPAR pathway modulation (Figure 3).

Most of the other plants that were tested showed low binding affinity for PPAR $\gamma$. Only grape wine (young shoots of Vitis vinifera) was more potent. Red wine was shown previously to be a potent PPAR $\gamma$ modulator and the red wine compounds ellagic acid and epicatechin gallate were identified as PPAR $\gamma$ ligands [13]. Wine or grape products have been also associated with other anti-diabetic effects; ethanolic wine grape pomace extract inhibited intenstinal $\alpha$-glucosidase and suppressed postprandial hyperglycemia in diabetic mice [17] and in humans, grape wine improved several metabolic responses such as decreased levels of blood glucose, insulin and glycated hemoglobin etc., which indicated better glycemic control and increased insulin sensitivity [18]. 
Citation: Medjakovic S, Hobiger S, Jungbauer A (2014) Pomegranate: High Binding Affinity for PPAR $\gamma$, a Drug Target for Diabetes Type 2, and Lipid Remodelling in Adipocytes. Nat Prod Chem Res 2 :136. doi:10.4172/2329-6836.1000136

Page 4 of 8

\begin{tabular}{|c|c|c|c|c|c|}
\hline Extract & Plant & Plant part & Internal product code (trade name) & Drug extract ratio & Additional information \\
\hline (1) & Pomegranate & Whole fruit & P8D & $75: 1$ & $80 \%$ Polyphenols \\
\hline (2) & Pomegranate & Fruit & PFD & $6: 1$ & $\begin{array}{c}70 \% \text { Polyphenols, } \\
55 \% \text { ellagitannins } \\
15-20 \% \text { ellagic acid, } \\
45 \% \text { punicalagins }\end{array}$ \\
\hline (3) & Pomegranate & Fruit & PSD & & $40 \%$ Ellagic acid \\
\hline$(4)$ & Pomegranate & Peel & PPD & $20: 1$ & $40 \%$ Ellagic acid \\
\hline$(5)$ & Pomegranate & Whole fruit & P7D & & $70 \%$ Ellagic acid \\
\hline (6) & Pomegranate & Not declared & PXD (Pomanox\$P20) & $40-50: 1$ & $\begin{array}{l}20 \% \text { Punicalagin } \alpha+\beta \text {, } \\
40 \% \text { total polyphenols }\end{array}$ \\
\hline$(7)$ & Grape wine & Young shoots & WRD (Vineatrol\$30) & & $30 \%$ Resveratrol \\
\hline (8) & Ginseng & Radix & GRD (EFLA\$910) & $5: 1$ & \\
\hline (9) & Kothala himbutu & Not declared & SRD (Exadipin\$) & 10:1 & \\
\hline$(10)$ & Purslane & Herb & POD & $7: 1$ & \\
\hline (11) & Bitter melon & Fruit & BMD & $10: 1$ & $10 \%$ Charantin \\
\hline (12) & Cinammon & Bark & ZZD & $10: 1$ & \\
\hline$(13)$ & Cinammon & Bark & ZFD & $15: 1$ & \\
\hline (14) & Ivy gourd & Not declared & CID (Coccinia D\$) & & \\
\hline
\end{tabular}

Table 1: Description and additional information of tested extracts.

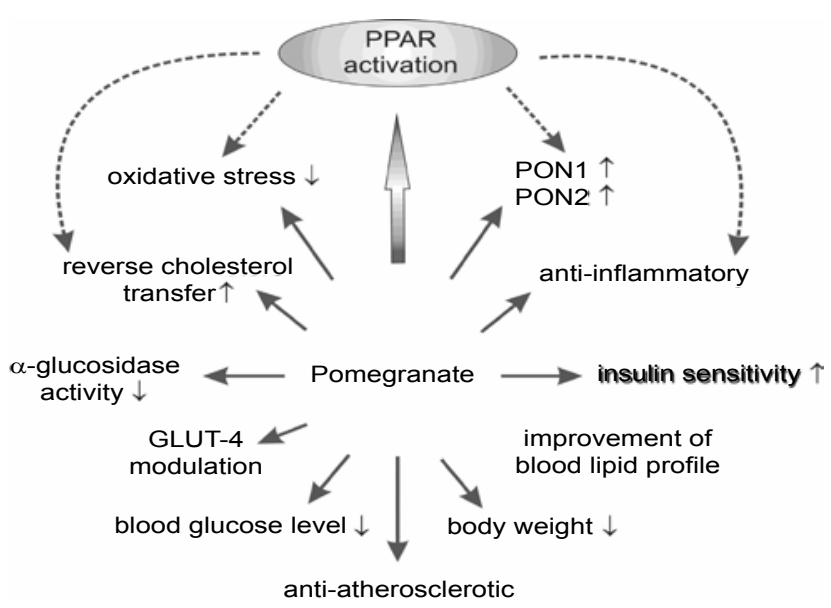

Figure 3: Overview of actions of pomegranate on hypoglycemia, insulin sensitivity, a-glucosidase inhibition, modulation of glucose transporter type 4 (GLUT4) function and blood lipid parameters. Several, but not all, of these actions can be linked to PPAR pathway modulation. Redrawn from Medjakovic and Jungbauer [45].

Ginseng (Panax ginseng C.A. Meyer) showed significant, albeit low, binding affinity for PPAR $\gamma$ in our test system. Ginsenoids, the bioactive compounds from ginseng, have been reported to be modulators of PPARa, $\beta$ and $\gamma$ [19-21]. PPAR pathways mediate several anti-diabetic actions or lipid metabolism affecting effects of these compounds, such as hypoglycemic effects [22-24], increased expression of GLUT4 $[23,25,26]$, increased expression of insulin receptor [26], decreased body weight (gain) [23,27], improvement of blood lipid parameters [28] and lipoprotein metabolism [23]. In spite of all the in vivo and in vitro data, a systematic review of randomized controlled trials, was not able to prove effectiveness of ginseng for diabetes type 2 treatment and the authors concluded with the appeal for more controlled studies [29].

The call for more clinical studies that investigate in a randomized and controlled way the effect of herbal or fruit supplements on diabetes is a common situation. This applies also for bitter melon (Momordica charantia), also called bitter gourd. This fruit has been in ethnomedical use in several countries for the treatment of diabetes and has been used for example for at least 600 years in traditional Chinese medicine as diabetes remedy [30]. In our test system, the binding affinity to PPAR $\gamma$ was barely detectable and relatively low. The study of Chao and Huang [31] report a moderate activation of PPAR $\alpha$ and $\gamma$ in a transactivation assay. More studies observed a modulation of PPAR $\alpha, \alpha$ and $\gamma$ expression [32-34], an increase in PPAR $\gamma$ phosphorylation [35] and increased PPAR $\alpha$ promoter activity [34]. The modulation of PPAR expression and protein function is potentially the underlying mechanism by which bitter melon regulates lipid and glucose metabolism and mediate anti-diabetic action, rather than the direct binding to PPARs and the stimulation of target gene transcription.

Kothala himbutu (Salacia reticulata) is another Ayurverdic medicinal plant that is widely used as diabetes remedy in Asia, together with other Salacia species such as Salacia oblonga. As Li, et al. reviewed, studies indicate several targets for Salacia in diabetes therapy; PPAR $\alpha$ activation and enzyme inhibition ( $\alpha$-glucosidase, pancreatic lipase, aldose reductase) were reported [36]. Our experiments showed only low binding affinity to PPAR $\gamma$.

Purslane (Portulaca oleracea) is part of the traditional Chinese medicine. Recently, dietary supplements have been marketed for the regulation of blood glucose levels. In this study, the binding affinity for PPAR $\gamma$ was low. In mice [37], but also in a small trial with humans [38], purslane had a significant benevolent effect on body weight, blood glucose and blood lipid parameters. But purslane has not been investigated intensively. Lee, et al. report also a suppression of diabetic vascular inflammation $[39,40]$.

Ivy gourd (Coccinia indica also known as Coccinia grandis or Coccinia cordifolia), grows abundantly in India and is an Ayurverdic medicinal plant used as diabetes treatment. In streptozotocin or alloxan induced diabetic rats, hypoglycemic, hypolipidemic and insulin elevating effects were reported [41-44]. In a placebo-controlled, randomized trial a significant decrease in fasting, postprandial blood glucose levels was observed. The mechanisms behind these effects are poorly investigated. A possible explanation is an inhibitory activity on a-amylase [45]. Nevertheless, as Coccinia indica was the only extract that showed no binding affinity for PPAR $\gamma$ in our assay, anti-diabetic effects via an activation of the PPAR $\gamma$ pathway are unlikely.

While ivy gourd has been barely researched, cinnamon has been investigated in depth. As one of the oldest spices, it is also part of Ayurvedic and Chinese medicine since hundreds or even thousands 
Citation: Medjakovic S, Hobiger S, Jungbauer A (2014) Pomegranate: High Binding Affinity for PPAR $\gamma$, a Drug Target for Diabetes Type 2, and Lipid Remodelling in Adipocytes. Nat Prod Chem Res 2 :136. doi:10.4172/2329-6836.1000136

of years. In this study, two extracts of the bark of Cinnamomum cassia, also known as Cinnamomum aromaticum or Chinese cinnamon, were tested. They showed low binding affinity for PPAR $\gamma$. The potential of cinnamon extract to bind to PPAR $\gamma$ [46], to transactivate PPARa and $\gamma$ [46], and increase PPAR $\alpha$ and $\gamma$ expression [47] was reported previously. Reported anti-diabetic properties of cinnamon include hypoglycemic, lipid-lowering and anti-inflammatory as well as insulin sensitivity increasing effects (reviewed $[48,49]$ ). The modulation of PPAR pathways is only one of several proposed mechanisms. The efficacy of cinnamon on diabetes in clinical trials remains uncertain; meta-analyses are inconsistent [50-52].

\section{Effect of pomegranate on adipocyte differentiation}

Pomegranate extract (2) had an extremely high binding affinity for

a)

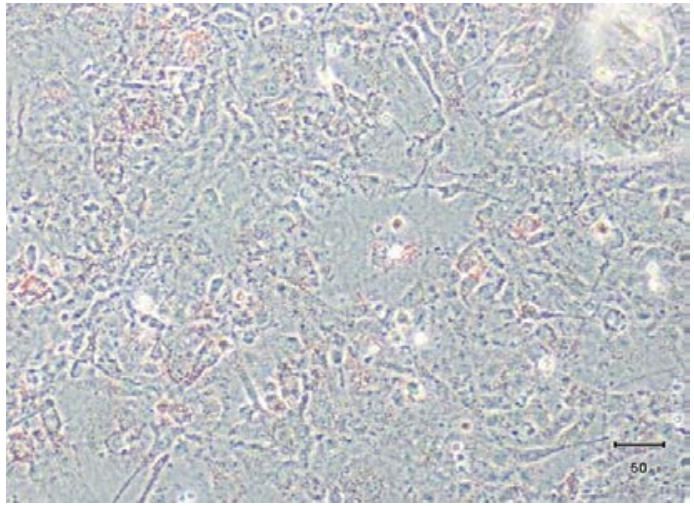

c)

d)

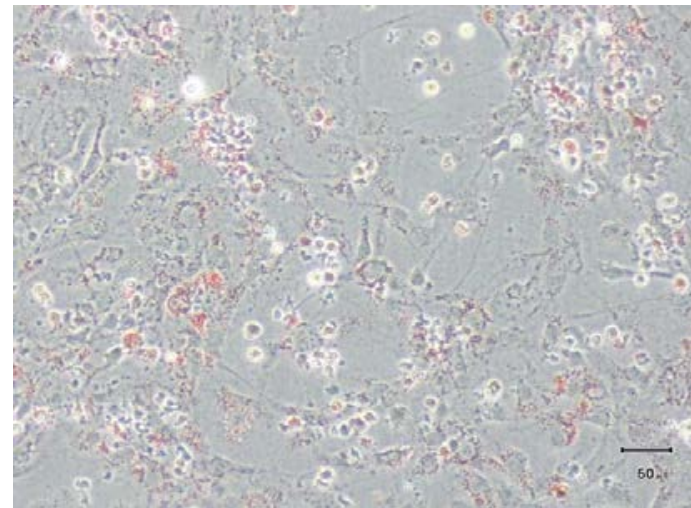

e)

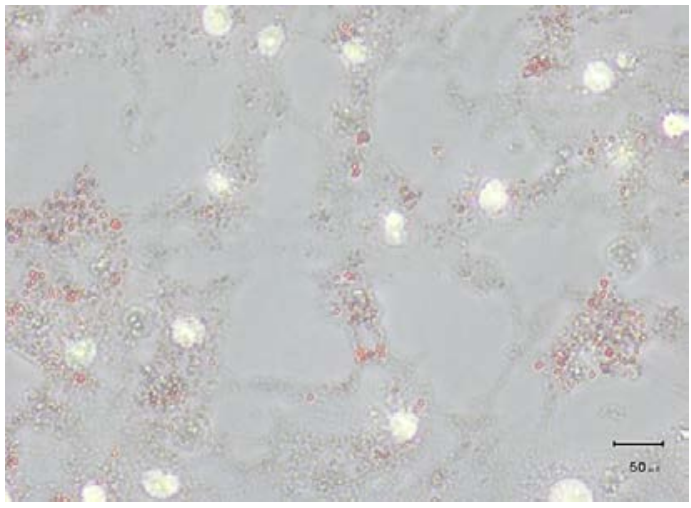

PPAR $\gamma$ and was selected to be tested in the adipocyte differentiation assay. After Oil Red O staining, it can be seen that upon induction of the differentiation with IBMX and DEX, followed by a treatment with progression media with insulin (Figure $4 \mathrm{~b}$ ), more Lipid Droplets (LDs) are stained compared to the basal level of the normal growth media (Figure 4a). The control with DMSO and with induction and progression media (Figure 4c) shows similar results to cells treated only with induction and progression media (Figure $4 \mathrm{~b}$ ). The addition of rosiglitazone increases the differentiation potently and leads also to the formation of very big LDs (Figure $4 \mathrm{~d}$ ). The addition of pomegranate extract to the induction and progression media leads to the formation of very small LDs (Figure 4e), compared to the control with only induction and progression media. This lipid remodeling and the formation of small droplets putatively enable an easier lipid breakdown in the lipolysis process.

b)
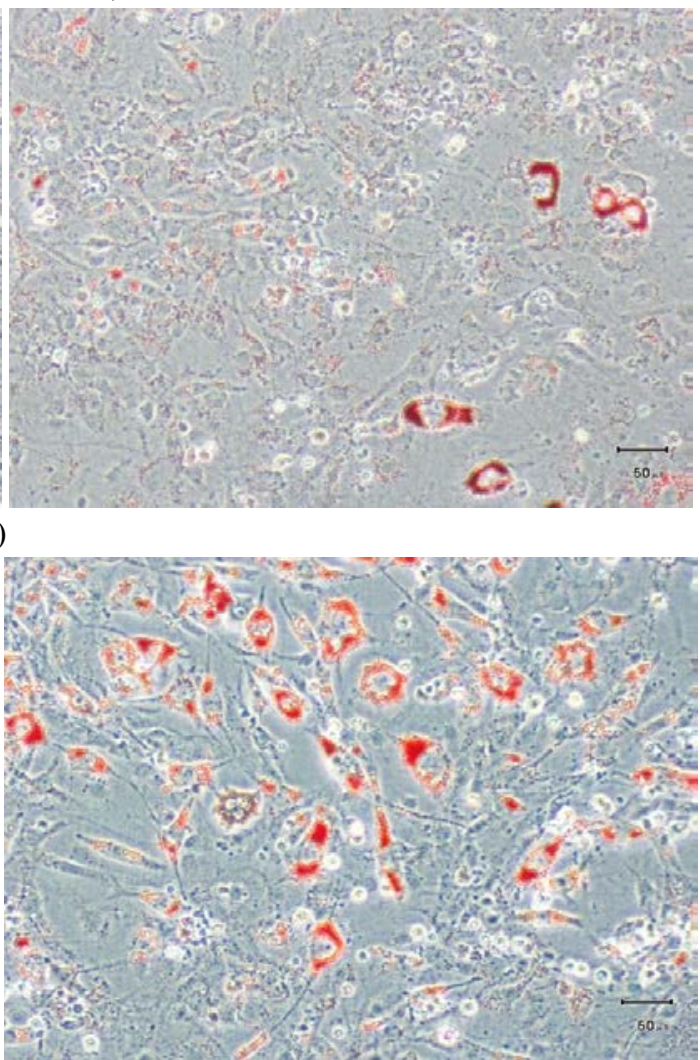

Figure 4: \% of inhibition of the Pentas lanceolata leaves. 
The measurement of the lipid droplets showed that upon rosiglitazone treatment, LDs were predominantly in the range of 6 to $11 \mu \mathrm{m}$ in diameter (Figure 5a). Meanwhile, cells that were treated only with FBS media were mostly below $3 \mu \mathrm{m}$ (Figure 5b). This was also true for cells treated with DMSO as vehicle (Figure 5c). Pomegranate extract, which was also extracted with DMSO, reduced lipid droplets mostly to $<1.5 \mu \mathrm{m}$ (Figure $5 \mathrm{~d}$ ). Most lipid droplet in cells treated with pomegranate extract, were too small for measurement with the size measurement function of the camera. They were $<1 \mu \mathrm{m}$ and can be defined as micro lipid droplets.

The formation of mLDs can be regarded as part or as facilitation of the lipolysis. Several just recently published studies suggest de novo synthesis of $\mathrm{mLDs}$, which become rapidly new sites of active lipolysis $[2,53]$.

Smaller LDs are faster degraded than large LDs [2]. The size of LDs is important for lipolysis efficiency: the higher the LD surface area, the more working surface lipases have and the lipolysis is more efficient. The lack of proteins, e.g. FSP27, that regulate the growth of LDs is associated with smaller LDs and increased lipolysis [54,55]. Upon activation of lipolysis, a fragmentation of large centrally located LDs can be observed; as consequence large LDs are absent and the cytoplasm is filled with mLDs [56].

mLD formation increases the total LD surface area by $\sim 30 \%$ but does not involve detectable fission from large LDs [53]. On the other hand, insulin stimulation of lipolytically active cells results in a decrease in mLDs and a concomitantly increase of large LDs (macro LDs) [53]. So, insulin inhibits lipolytic signalling and promotes lipid storage [53]. The promotion of lipid storage is mediated by an increase in the reesterification of free fatty acids [57].

In this study, we see that compared to cells that are treated only with media (induction media, followed by progression media); no big LDs are generated upon treatment with pomegranate extract. In regard of the above mentioned and discussed studies, the formation of finely dispersed mLDs in the adipocytes after pomegranate treatment, suggest the activation of lipolysis. This could explain experiments with mice that link pomegranate to decreased body weight, although energy intake was unaffected $[58,59]$. It could also explain why in those very same studies, a decrease in body fat mass, but no alterations in lean mass [59], or no significant differences in absolute fat mass [58] were observed, despite the impact on body weight.

\section{Conclusion}

The activation of lipolysis rounds out the picture of pomegranate as anti-diabetic fruit that ameliorates symptoms of metabolic syndrome. Pomegranate as PPAR $\gamma$ modulator was described previously, but the extremely higher binding affinity for PPAR $\gamma$ compared to plant extracts that are commercially offered as anti-diabetics, is a new revelation. a)

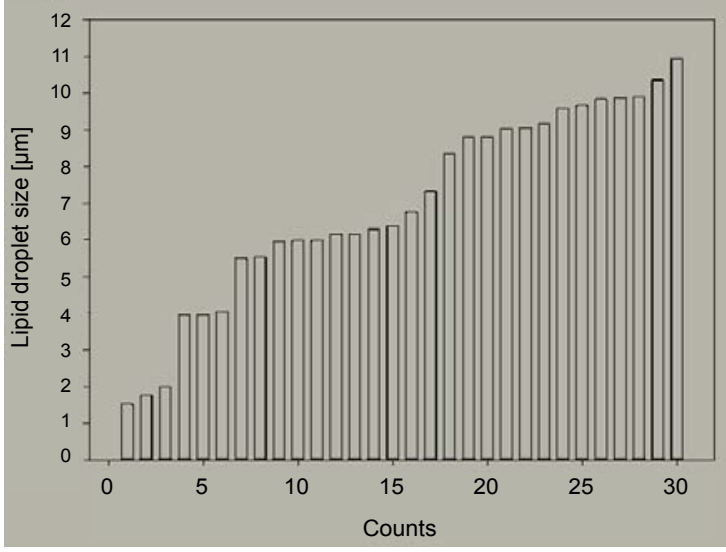

c)

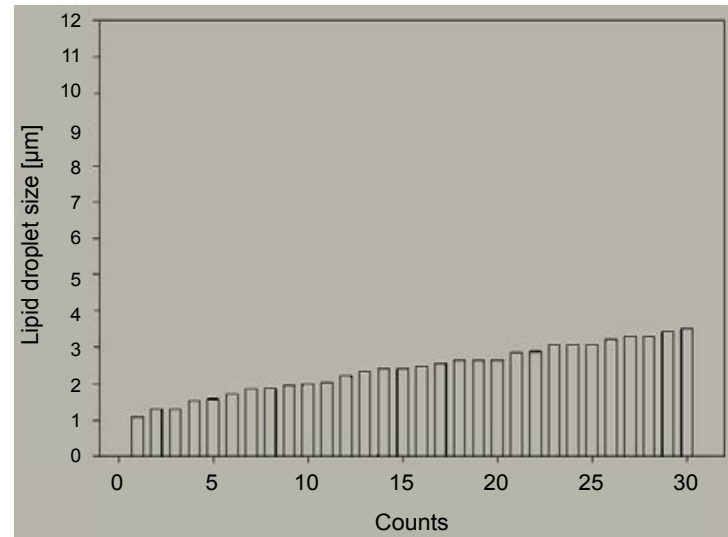

b)

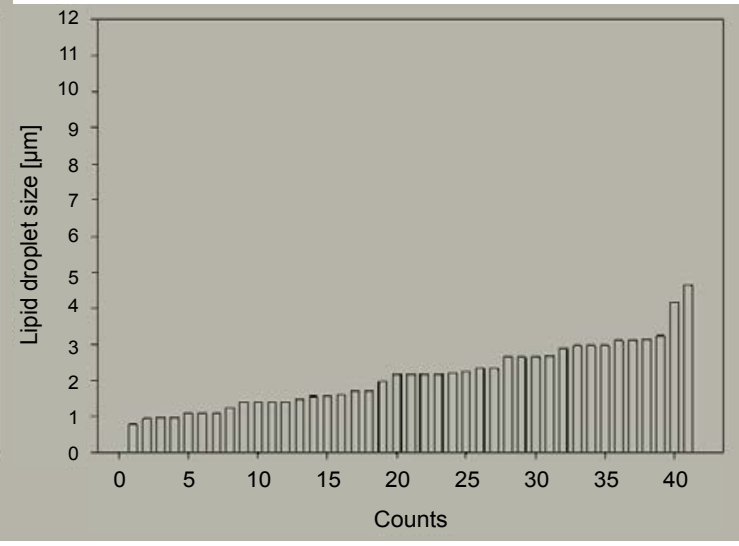

d)

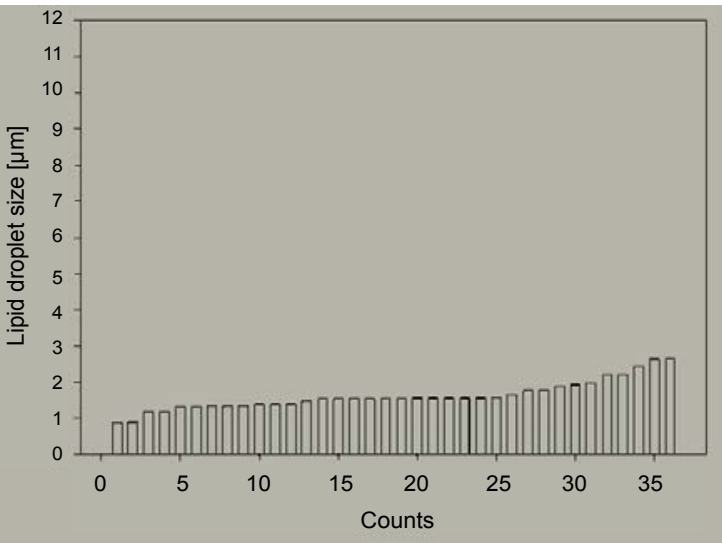

Figure 5: Size distribution of lipid droplets after adipocyte differentiation. Pictures show the effect upon treatment of a) rosiglitazone (final concentration $0.1 \mu \mathrm{M}$ in DMSO), b) control, growth media with FBS, c) control, DMSO, and d) extract (2) pomegranate fruit in DMSO. Microscopic pictures of a representative adipocyte differentiation assay after Oil Red $O$ staining on day 9 (total of 11 days of incubation). 
The effects of pomegranate that are reported in this study, explain in no way all of the reported in vivo and in vitro results that attribute pomegranate anti-diabetic properties, but they provide more puzzle pieces that complement the picture.

Beside the medication of people suffering from metabolic syndrome, life style changes that can prevent obesity, diabetes and metabolic syndrome are needed. One possibility is the change of diet to more healthy food and even better, the consumption of food that may even oppose effects of unhealthy food. Pomegranate is a good candidate for that. Several studies link pomegranate to hypoglycemic effects, an improvement of insulin sensitivity and blood lipid profile, as well as anti-inflammatory effects. Moreover, the fruit is tasty, so it is not a problem to include it in the normal diet as the acceptance of people of all ages is given. Due the very long shelf-life of the fruit and the possibility to use the fruit juice, a worldwide distribution poses also no problem. The well-known saying: "An apple a day, keeps the doctor away!" could be applied to pomegranate.

But pomegranate can be used on different levels for prevention, amelioration and treatment of diabetes and metabolic syndrome. In the first place, pomegranate as fruit or juice has the potential to be a preventive measure that can be easily included in the normal diet. Secondly, dietary supplements with pomegranate can also be used as co-treatment along with the traditional medicine; about $30 \%$ of diabetics use already frequently [60-62] complementary and alternative medicine supplements to treat their diabetes together with the prescribed diabetes medication. Our in vitro results show that 100 $\mathrm{mg}$ of the most potent extract (P8D) could be effective as co-treatment and a good start for in vivo evaluation. And last but not least, is the isolation of bioactive compounds from pomegranate that have antidiabetic properties for the implementation as drug, also a possibility; metformin has also been developed from French lilac, which was used for a long time as herbal anti-diabetic. Especially in the antidiabetic drug class of PPAR $\gamma$ modulators there is potential need, as the currently used glitazones have been withdrawn from the market or are hotly debated because of possible side effects.

There is certainly a need for more randomized and controlled clinical trials with a good study design to verify all the in vitro and in vivo data and the common use of pomegranate in ethnomedicine for the treatment of diabetes, obesity and metabolic syndrome. Regardless of that nutritionist should recommend the consumption of pomegranate as part of a healthy diet.

\section{References}

1. Listenberger LL, Han X, Lewis SE, Cases S, Farese RV Jr, et al. (2003) Triglyceride accumulation protects against fatty acid-induced lipotoxicity. Proc Natl Acad Sci U S A 100: 3077-3082.

2. Paar M, Jüngst $C$, Steiner NA, Magnes $C$, Sinner F, et al. (2012) Remodeling of lipid droplets during lipolysis and growth in adipocytes. J Biol Chem 287: 11164-11173.

3. Hashimoto T, Segawa H, Okuno M, Kano H, Hamaguchi HO, et al. (2012) Active involvement of micro-lipid droplets and lipid-droplet-associated proteins in hormone-stimulated lipolysis in adipocytes. J Cell Sci 125: 6127-6136.

4. (2012) Obesity and overweight. World Health Organization.

5. Mazzola N (2012) Review of current and emerging therapies in type 2 diabetes mellitus. Am J Manag Care 18: S17-26.

6. Lewis JD, Ferrara A, Peng T, Hedderson M, Bilker WB, et al. (2011) Risk of bladder cancer among diabetic patients treated with pioglitazone: interim report of a longitudinal cohort study. Diabetes Care 34: 916-922.

7. Neumann A, Weill A, Ricordeau P, Fagot JP, Alla F, et al. (2012) Pioglitazone and risk of bladder cancer among diabetic patients in France: a populationbased cohort study. Diabetologia 55: 1953-1962.
8. Tenenbaum A, Motro M, Fisman EZ (2005) Dual and pan-peroxisome proliferator-activated receptors (PPAR) co-agonism: the bezafibrate lessons. Cardiovasc Diabetol 4: 14.

9. Bassaganya-Riera J, DiGuardo M, Climent M, Vives C, Carbo A, et al. (2011) Activation of PPARy and $\delta$ by dietary punicic acid ameliorates intestinal inflammation in mice. Br J Nutr 106: 878-886.

10. Hontecillas R, O'Shea M, Einerhand A, Diguardo M, Bassaganya-Riera $\mathrm{J}$ (2009) Activation of PPAR gamma and alpha by punicic acid ameliorates glucose tolerance and suppresses obesity-related inflammation. J Am Coll Nutr 28: 184-195.

11. Li Y, Qi Y, Huang TH, Yamahara J, Roufogalis BD (2008) Pomegranate flower: a unique traditional antidiabetic medicine with dual PPAR-alpha/-gamma activator properties. Diabetes Obes Metab 10: 10-17.

12. Shiner M, Fuhrman B, Aviram M (2007) Macrophage paraoxonase 2 (PON2) expression is up-regulated by pomegranate juice phenolic anti-oxidants via PPAR gamma and AP-1 pathway activation. Atherosclerosis 195: 313-321.

13. Zoechling A, Liebner F, Jungbauer A (2011) Red wine: a source of poten ligands for peroxisome proliferator-activated receptor $\hat{i}^{3}$. Food Funct 2: 28-38.

14. Kohno H, Suzuki R, Yasui Y, Hosokawa M, Miyashita K, et al. (2004) Pomegranate seed oil rich in conjugated linolenic acid suppresses chemically induced colon carcinogenesis in rats. Cancer Sci 95: 481-486.

15. Mueller M, Jungbauer A (2009) Culinary plants, herbs and spices - A rich source of PPARg ligands. Food Chemistry 117: 660-667.

16. Medjakovic S, Jungbauer A (2013) Pomegranate: a fruit that ameliorates metabolic syndrome. Food Funct 4: 19-39.

17. Hogan S, Zhang L, Li J, Sun S, Canning C, et al. (2010) Antioxidant rich grape pomace extract suppresses postprandial hyperglycemia in diabetic mice by specifically inhibiting alpha-glucosidase. Nutr Metab (Lond) 7: 71.

18. Banini AE, Boyd LC, Allen JC, Allen HG, Sauls DL (2006) Muscadine grape products intake, diet and blood constituents of non-diabetic and type 2 diabetic subjects. Nutrition 22: 1137-1145.

19. Chen LM, Lin ZY, Zhu YG, Lin N, Zhang J, et al. (2012) Ginsenoside Rg1 attenuates $\beta$-amyloid generation via suppressing PPAR $\gamma$-regulated BACE1 activity in N2a-APP695 cells. Eur J Pharmacol 675: 15-21.

20. Kwok HH, Yue PY, Mak NK, Wong RN (2012) Ginsenoside Rb induces type I collagen expression through peroxisome proliferator-activated receptor-delta. Biochem Pharmacol 84: 532-539.

21. Lee H, Gonzalez FJ, Yoon M (2006) Ginsenoside Rf, a component of ginseng, regulates lipoprotein metabolism through peroxisome proliferator-activated receptor alpha. Biochem Biophys Res Commun 339: 196-203.

22. Chung SH, Choi CG, Park SH (2001) Comparisons between white ginseng radix and rootlet for antidiabetic activity and mechanism in KKAy mice. Arch Pharm Res 24: 214-218.

23. Lim S, Yoon JW, Choi SH, Cho BJ, Kim JT, et al. (2009) Effect of ginsam, vinegar extract from Panax ginseng, on body weight and glucose homeostasis in an obese insulin-resistant rat model. Metabolism 58: 8-15.

24. Park MY, Lee KS, Sung MK (2005) Effects of dietary mulberry, Korean red ginseng, and banaba on glucose homeostasis in relation to PPAR-alpha, PPAR-gamma, and LPL mRNA expressions. Life Sci 77: 3344-3354.

25. Han KL, Jung MH, Sohn JH, Hwang JK (2006) Ginsenoside 20S-protopanaxatrio (PPT) activates peroxisome proliferator-activated receptor gamma (PPARgamma) in 3T3-L1 adipocytes. Biol Pharm Bull 29: 110-113.

26. Mollah ML, Kim GS, Moon HK, Chung SK, Cheon YP, et al. (2009) Antiobesity effects of wild ginseng (Panax ginseng C.A. Meyer) mediated by PPARgamma, GLUT4 and LPL in ob/ob mice. Phytother Res 23: 220-225.

27. Kim HJ, Kang HJ, Seo JY, Lee CH, Kim YS, et al. (2011) Antiobesity effect of oil extract of ginseng. J Med Food 14: 573-583.

28. Yoon M, Lee H, Jeong S, Kim JJ, Nicol CJ, et al. (2003) Peroxisome proliferatoractivated receptor alpha is involved in the regulation of lipid metabolism by ginseng. Br J Pharmacol 138: 1295-1302.

29. Kim S, Shin BC, Lee MS, Lee H, Ernst E (2011) Red ginseng for type 2 diabetes mellitus: a systematic review of randomized controlled trials. Chin J Integr Med 17: 937-944. 
Citation: Medjakovic S, Hobiger S, Jungbauer A (2014) Pomegranate: High Binding Affinity for PPAR $\gamma$, a Drug Target for Diabetes Type 2, and Lipid Remodelling in Adipocytes. Nat Prod Chem Res 2 :136. doi:10.4172/2329-6836.1000136

Page 8 of 8

30. Yin J, Zhang $\mathrm{H}$, Ye J (2008) Traditional chinese medicine in treatment of metabolic syndrome. Endocr Metab Immune Disord Drug Targets 8: 99-111.

31. Chao CY, Huang CJ (2003) Bitter gourd (Momordica charantia) extract activates peroxisome proliferator-activated receptors and upregulates the expression of the acyl CoA oxidase gene in H4IIEC3 hepatoma cells. J Biomed Sci 10: 782-791.

32. Chao CY, Yin MC, Huang CJ (2011) Wild bitter gourd extract up-regulates mRNA expression of PPARa, PPAR $\gamma$ and their target genes in C57BL/6J mice. J Ethnopharmacol 135: 156-161.

33. Gadang V, Gilbert W, Hettiararchchy N, Horax R, Katwa L, et al. (2011) Dietary bitter melon seed increases peroxisome proliferator-activated receptor- $\gamma$ gene expression in adipose tissue, down-regulates the nuclear factor- $\kappa \mathrm{B}$ expression, and alleviates the symptoms associated with metabolic syndrome. J Med Food 14: 86-93.

34. Sasa M, Inoue I, Shinoda Y, Takahashi S, Seo M, et al. (2009) Activating effect of momordin, extract of bitter melon (Momordica Charantia L.), on the promoter of human PPARdelta. J Atheroscler Thromb 16: 888-892.

35. Chou YC, Su HM, Lai TW, Chyuan JH, Chao PM (2012) cis-9, trans-11, trans-13-Conjugated linolenic acid induces apoptosis and sustained ERK phosphorylation in 3T3-L1 preadipocytes. Nutrition 28: 803-811.

36. Li Y, Huang TH, Yamahara J (2008) Salacia root, a unique Ayurvedic medicine, meets multiple targets in diabetes and obesity. Life Sci 82: 1045-1049.

37. Gong F, Li F, Zhang L, Li J, Zhang Z, et al. (2009) Hypoglycemic effects of crude polysaccharide from Purslane. Int J Mol Sci 10: 880-888.

38. El-Sayed M (2011) Effects of Portulaca oleracea L. seeds in treatment of type-2 diabetes mellitus patients as adjunctive and alternative therapy. J Ethnopharmacol 137: 643-651.

39. Lee AS, Lee YJ, Lee SM, Yoon JJ, Kim JS, et al. (2012) An aqueous extract of Portulaca oleracea ameliorates diabetic nephropathy through suppression of renal fibrosis and inflammation in diabetic $\mathrm{db} / \mathrm{db}$ mice. Am J Chin Med 40: 495-510.

40. Lee AS, Lee YJ, Lee SM, Yoon JJ, Kim JS, et al. (2012) Portulaca oleracea Ameliorates Diabetic Vascular Inflammation and Endothelial Dysfunction in $\mathrm{db}$ db Mice. Evid Based Complement Alternat Med 2012: 741824.

41. Kar A, Choudhary BK, Bandyopadhyay NG (2003) Comparative evaluation of hypoglycaemic activity of some Indian medicinal plants in alloxan diabetic rats. J Ethnopharmacol 84: 105-108.

42. Mallick C, Chatterjee K, Guhabiswas M, Ghosh D (2007) Antihyperglycemic effects of separate and composite extract of root of Musa paradisiaca and leaf of Coccinia indica in streptozotocin-induced diabetic male albino rat. Afr J Tradit Complement Altern Med 4: 362-371.

43. Manjula S, Ragavan B (2007) Hypoglycemic and Hypolipidemic effect of Coccinia indica Wight \& Arn in alloxan induced diabetic rats. Anc Sci Life 27: 34-37.

44. Pari L, Venkateswaran S (2003) Protective effect of Coccinia indica on changes in the fatty acid composition in streptozotocin induced diabetic rats. Pharmazie 58: 409-412.

45. P S, Zinjarde SS, Bhargava SY, Kumar AR (2011) Potent a-amylase inhibitory activity of Indian Ayurvedic medicinal plants. BMC Complement Altern Med 11: 5

46. Sheng X, Zhang Y, Gong Z, Huang C, Zang YQ (2008) Improved Insulin Resistance and Lipid Metabolism by Cinnamon Extract through Activation of Peroxisome Proliferator-Activated Receptors. PPAR Res 2008: 581348.

47. Kim SH, Choung SY (2010) Antihyperglycemic and antihyperlipidemic action of Cinnamomi Cassiae (Cinnamon bark) extract in C57BL/Ks db/db mice. Arch Pharm Res 33: 325-333.
48. Bandara T, Uluwaduge I, Jansz ER (2012) Bioactivity of cinnamon with special emphasis on diabetes mellitus: a review. Int J Food Sci Nutr 63: 380-386.

49. Qin B, Panickar KS, Anderson RA (2010) Cinnamon: potential role in the prevention of insulin resistance, metabolic syndrome, and type 2 diabetes. $J$ Diabetes Sci Technol 4: 685-693.

50. Akilen R, Tsiami A, Devendra D, Robinson N (2012) Cinnamon in glycaemic control: Systematic review and meta analysis. Clin Nutr 31: 609-615.

51. Baker WL, Gutierrez-Williams G, White CM, Kluger J, Coleman Cl (2008) Effect of cinnamon on glucose control and lipid parameters. Diabetes Care 31: 41-43.

52. Leach MJ, Kumar S (2012) Cinnamon for diabetes mellitus. Cochrane Database Syst Rev 9: CD007170.

53. Ariotti N, Murphy S, Hamilton NA, Wu L, Green K, et al. (2012) Postlipolytic insulin-dependent remodeling of micro lipid droplets in adipocytes. Mol Biol Cell 23: $1826-1837$

54. Keller P, Petrie JT, De Rose P, Gerin I, Wright WS, et al. (2008) Fat-specific protein 27 regulates storage of triacylglycerol. J Biol Chem 283: 14355-14365.

55. Puri V, Konda S, Ranjit S, Aouadi M, Chawla A, et al. (2007) Fat-specific protein 27 , a novel lipid droplet protein that enhances triglyceride storage. $J$ Biol Chem 282: 34213-34218.

56. Marcinkiewicz A, Gauthier D, Garcia A, Brasaemle DL (2006) The phosphorylation of serine 492 of perilipin a directs lipid droplet fragmentation and dispersion. J Biol Chem 281: 11901-11909.

57. Campbell PJ, Carlson MG, Hill JO, Nurjhan N (1992) Regulation of free fatty acid metabolism by insulin in humans: role of lipolysis and reesterification. Am J Physiol 263: E1063-1069.

58. McFarlin BK, Strohacker KA, Kueht ML (2009) Pomegranate seed oil consumption during a period of high-fat feeding reduces weight gain and reduces type 2 diabetes risk in CD-1 mice. Br J Nutr 102: 54-59.

59. Vroegrijk IO, van Diepen JA, van den Berg S, Westbroek I, Keizer H, et al (2011) Pomegranate seed oil, a rich source of punicic acid, prevents dietinduced obesity and insulin resistance in mice. Food Chem Toxicol 49: 14261430

60. Bradley R, Sherman KJ, Catz S, Calabrese C, Jordan L, et al. (2011) Survey of CAM interest, self-care, and satisfaction with health care for type 2 diabetes at group health cooperative. BMC Complement Altern Med 11: 121.

61. Fabian E, Töscher S, Elmadfa I, Pieber TR (2011) Use of complementary and alternative medicine supplements in patients with diabetes mellitus. Ann Nutr Metab 58: 101-108.

62. Manya K, Champion B, Dunning T (2012) The use of complementary and alternative medicine among people living with diabetes in Sydney. BMC Complement Altern Med 12: 2. 